\title{
IDENTIFICATION OF HUMAN SPERM-COATING ANTIGEN
}

\author{
T. K. ROBERTS AND B. BOETTCHER \\ School of Biological Sciences, The Flinders University of South Australia, \\ Bedford Park, South Australia 5042
}

(Received 2nd August 1968, accepted 30th October 1968)

Summary. SGA has been identified as an iron-binding protein present in seminal plasma. It has an electrophoretic mobility similar to transferrin, and different from lactoferrin, whereas it shares immunological characteristics with lactoferrin not possessed by transferrin. The name scaferrin is proposed for this compound.

In 1956 Weil, Kotsevalov \& Wilson showed that antisera to whole human semen, seminal plasma and washed seminal spermatozoa were indistinguishable in specificity, and were directed against an antigenic component of seminal plasma. Further work has established that the most antigenic component of seminal spermatozoa is adsorbed on to them from the seminal plasma. Weil has termed this component of seminal plasma, sperm-coating antigen (SCA).

The known properties of SCA are as follows:

(a) It originates in the seminal vesicle (Weil \& Rodenburg, 1962).

(b) It is the most antigenic component of seminal spermatozoa when these are injected into heterologous animals (Weil, 1965).

(c) It cannot be detected on testicular or epididymal spermatozoa with antisera against seminal spermatozoa (Weil \& Rodenburg, 1960).

(d) The same antigen is present in seminal plasma, even in the absence of spermatozoa, such as in azoospermic ejaculates (Weil et al., 1956).

(e) It is a $\beta$-globulin of large molecular size; it is non-dialysable; and its antigenic properties are destroyed by boiling (Weil, 1967).

We have injected washed human seminal spermatozoa into rabbits. Two 1-ml intramuscular injections, of a 1:1 mixture of packed, washed spermatozoa in Freund's complete adjuvant, were found to be sufficient to induce the rabbit to produce antibodies to homogenized seminal spermatozoa. A similar procedure has been used to produce antibodies to seminal plasma.

The antiserum to washed seminal spermatozoa (anti-sperm) produced a single line in immunodiffusion against normal specimens of seminal plasma and homogenized spermatozoa, but did not produce precipitation with samples of serum, or with homogenates of salivary gland, pancreas and liver (Table 1; PI. 1, Fig. 1). The antiserum to seminal plasma showed similar specificity.

Immunoelectrophoresis of seminal plasma was performed against the antisperm serum, and the anti-human seminal plasma serum. Single lines of 
precipitation were produced by both antisera, and these appeared to be identical (Pl. 1, Fig. 2). The activity of these antisera against seminal plasma could be removed by absorption with washed seminal spermatozoa.

The anti-sperm antiserum described here appears to be directed against SGA. It was produced by injecting washed human seminal spermatozoa into rabbits; it recognizes the most antigenic component of washed seminal spermatozoa; it reacts against seminal spermatozoa and seminal plasma identically; and it has specificity identical with an anti-seminal plasma antiserum.

In attempts to identify the component of seminal plasma against which the anti-sperm antiserum is directed, immunoelectrophoresis of human seminal plasma against the anti-sperm antiserum was performed. In one case the seminal

TABLE 1

IMMUNODIFFUSION OF ANTI-SPERM ANTISERUM AGAINST VARIOUS SAMPLES

\begin{tabular}{l|c|c}
\hline \multicolumn{1}{c|}{ Sample } & $\begin{array}{c}\text { No. } \\
\text { tested }\end{array}$ & $\begin{array}{c}\text { No. giving } \\
\text { precipitation }\end{array}$ \\
\hline Seminal plasma & 33 & 33 \\
Whole semen & 6 & 6 \\
Azoospermic semen & 2 & 2 \\
Serum & 3 & 0 \\
Salivary gland homogenate & 1 & 0 \\
Pancreas homogenate & 1 & 0 \\
Liver homogenate & 1 & 0 \\
Homogenized seminal spermatozoa & 2 & 2 \\
Dialysed seminal plasma & 2 & 2 \\
Epididymis homogenate & 1 & 0 \\
Testis homogenate & 1 & 0 \\
-Spermatocoele fluid & 1 & 0 \\
& & \\
\hline
\end{tabular}

plasma had been pre-incubated with $\left[{ }^{59} \mathrm{Fe}\right]$ ferric citrate. (To label the seminal plasma with ${ }^{59} \mathrm{Fe}, 1$ drop of radio-active ferric citrate, approximately $0.6 \mu \mathrm{c}$, was dried in a small test-tube and 2 drops of seminal plasma were added. The mixture was incubated at $25^{\circ} \mathrm{C}$ for $15 \mathrm{~min}$. Immunoelectrophoresis of the labelled seminal plasma produced a single precipitation arc, and this, on autoradiography, was found to be radio-active ( $\mathrm{Pl}$. 1, Fig. 3). Autoradiography of immunodiffusion tests involving the anti-sperm antiserum and ${ }^{59} \mathrm{Fe}$-labelled seminal plasma confirmed that the single component of seminal plasma recognized by the antiserum is an iron-binding protein.

It is concluded that SGA is an iron-binding protein found in seminal plasma.

\section{EXPLANATION OF PLATE 1}

FIG. 1 (top). Immunodiffusion of anti-sperm antiserum (centre well) against serum (1), seminal plasma $(2,4,6)$ and washed seminal spermatozoa homogenate $(3,5)$. Serum gives no precipitate, whereas seminal plasma and spermatozoal homogenate give a single line of precipitation.

Fig. 2 (middle). Immunoelectrophoresis of human seminal plasma against anti-sperm antiserum (lower trough) and anti-seminal plasma antiserum (upper trough). Identical lines of precipitation with both antisera can be seen.

FIG. 3 (bottom). (a) Immunoelectrophoresis of seminal plasma (lower well) and serum (upper well) against anti-sperm antiserum. Both specimens have been labelled with ${ }_{59} \mathrm{Fe}$. (b) Autoradiograph of the same slide. The line of precipitation between seminal plasma and antisperm is seen to be radio-active. 


\section{PLATE 1}

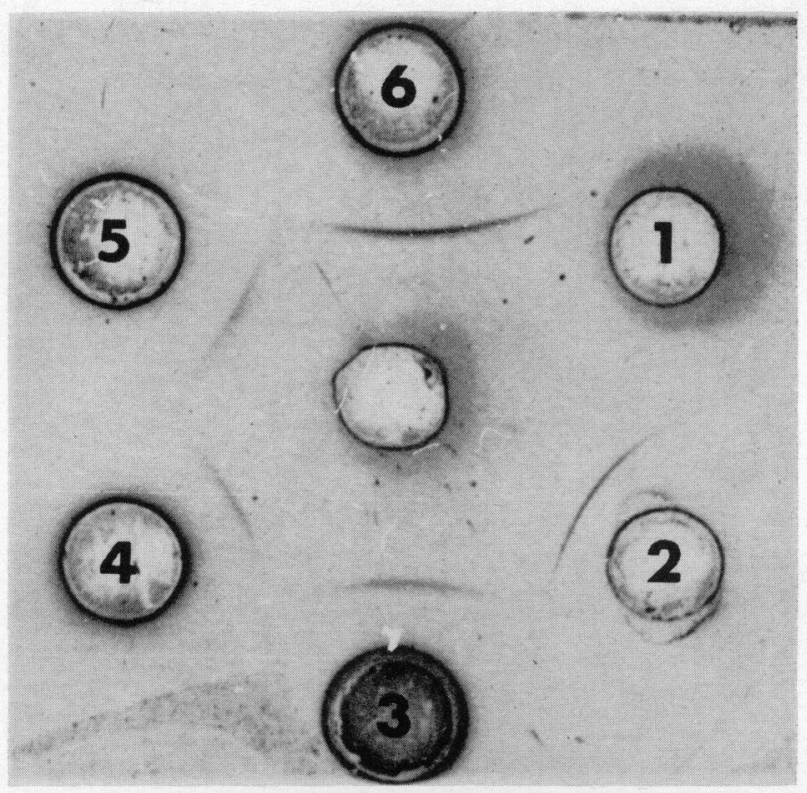

$$
\text { anti-SP }
$$

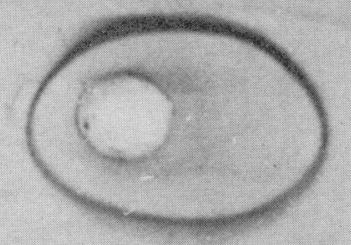

$$
\text { ant } \mathrm{i}-\mathrm{sperm}
$$

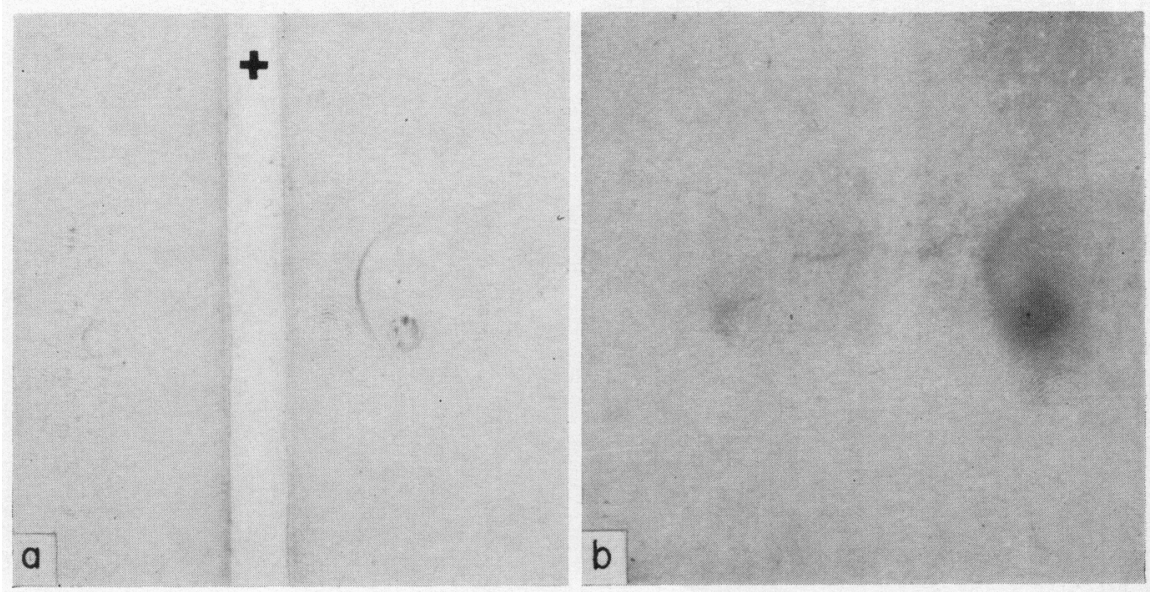


PLATE 2
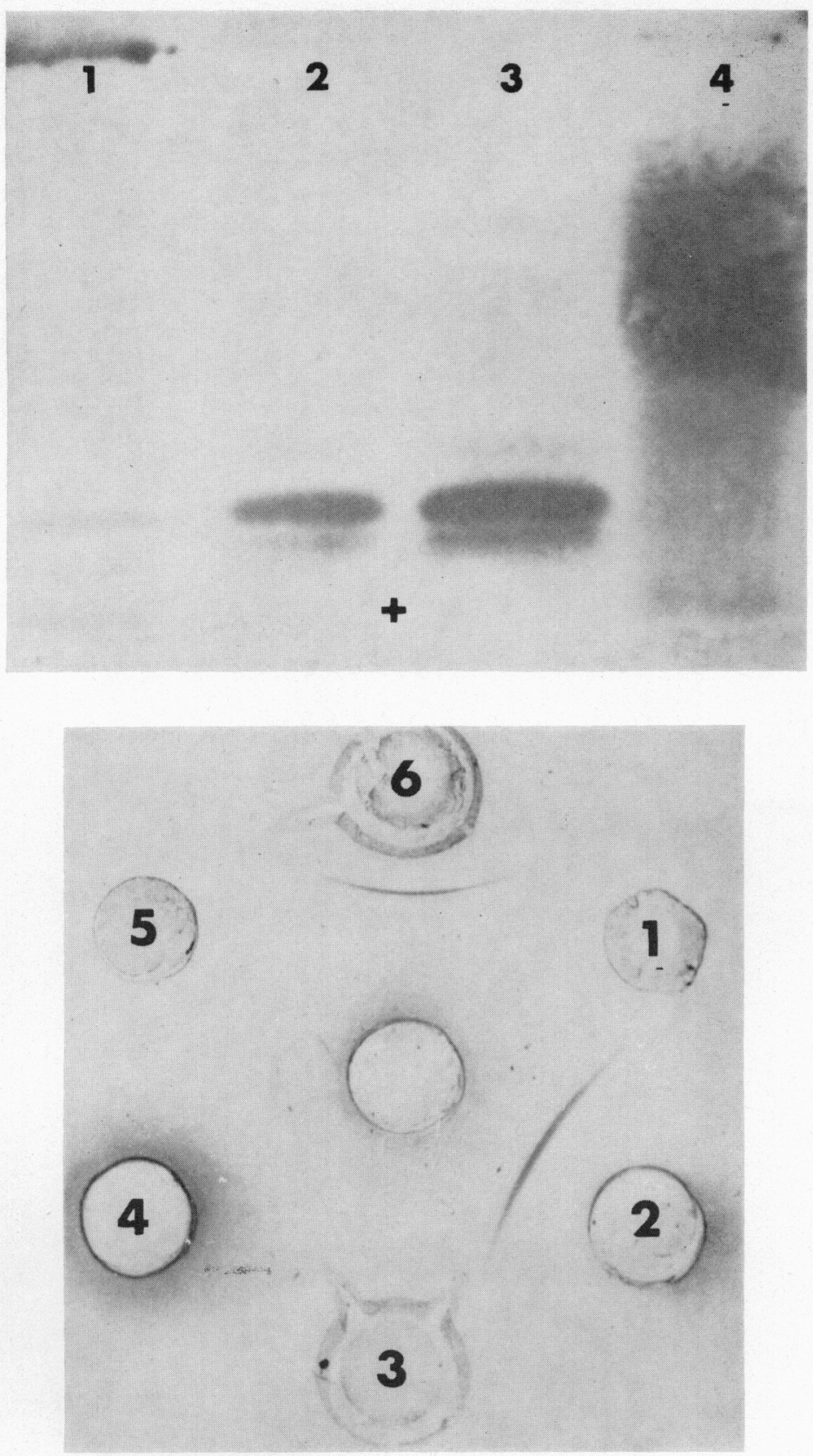

(Facing p. 349) 
Starch gel electrophoresis, followed by autoradiography, was performed using seminal plasmas, human serum and human lactoferrin (isolated by the technique of Masson \& Heremans, 1966), labelled with ${ }^{59} \mathrm{Fe}$, by a procedure similar to that given above (Pl. 2, Fig. 4). The autoradiographs revealed a single zone of radio-activity in the seminal plasma in a position corresponding to the zone of activity shown by serum transferrin (in the $\beta$-globulin region), and different from the zone of radio-activity in the lactoferrin sample. This indicates that in seminal plasma there is a single species of iron-binding protein and that this migrates under electrophoresis with a mobility similar to that of serum transferrin. This mobility is different from that of lactoferrin.

Under the conditions of electrophoresis employed, lactoferrin showed a broad zone of activity, whereas seminal plasma and serum showed well-defined zones. Szuchet-Derechin \& Johnson (1966) subjected bovine serum transferrin and lactoferrin to electrophoresis and found, similarly, that they have different mobilities, and that, under conditions where transferrin bands are well resolved, lactoferrin produces a broad zone.

Immunodiffusion of the anti-sperm antiserum against seminal plasmas, human serum and human lactoferrin, showed that it has similar activity against seminal plasma and lactoferrin, but no activity against serum (Table 1; PI. 2, Fig. 5).

Consequently, it appears that SGA has an electrophoretic mobility similar to serum transferrin, and different from lactoferrin, though it has some immunological properties which are possessed by lactoferrin but not by transferrin. Further evidence of immunological differences between serum transferrin and SCA has been obtained since some seminal plasmas, which would not give precipitates in either immunodiffusion or immunoelectrophoresis with horse anti-transferrin (Hyland, lot no. 8415 D001A1), showed iron-binding activity or autoradiography after starch gel electrophoresis and, also, gave precipitation with the anti-sperm antiserum on immunodiffusion.

Since we have now identified SCA as an iron-binding protein in seminal plasma, which is not identical with either transferrin or lactoferrin, we propose that it be termed 'scaferrin', for obvious reasons.

Recently, Hekman \& Rumke (1968) have shown that one of the antigenic components in human seminal plasma has immunological properties in common with lactoferrin and, in fact, they identified it as lactoferrin. Further, they showed that it was present on seminal spermatozoa, but absent from testis,

\section{EXPLANATION OF PLATE 2}

Fig. 4 (above). Autoradiograph of starch gel electrophoretogram of seminal plasma (samples 1 and 2) serum (sample 3) and lactoferrin preparation (sample 4) labelled with ${ }^{59} \mathrm{Fe}$. Sample application slots are near the top of the figure. The buffer front is near the bottom of the figure. ( $12 \%$ starch gel. Electrolyte buffer $0.029 \mathrm{M}-\mathrm{LiOH}, 0.165 \mathrm{M}$-boric acid, $\mathrm{pH} 8.5$. Gel buffer $0.019 \mathrm{~m}$-tris, $0.0178 \mathrm{~m}$-cacodylic acid, $\mathrm{pH} 7 \cdot 3$.) The iron-binding protein in seminal plasma has an electrophoretic mobility similar to serum transferrin, but different from that of lactoferrin.

FIG. 5 (below). Immunodiffusion of anti-sperm antiserum (centre well) against serum (1), lactoferrin (2), homogenate of epididymis (3), homogenate of testis (4), spermatocoele fluid $(5)$ and seminal plasma $(6)$. The anti-sperm antiserum gives a single line of precipitation with only lactoferrin and seminal plasma. 
epididymal extract and testicular spermatozoa. It seems likely that the compound studied by them is scaferrin.

The physiological role of SCA is not yet established (Weil, 1967). Any proposed role for this substance must take into account the observation that SGA has only been shown to be antigenic on seminal spermatozoa when these are injected into heterologous animals (Weil \& Finkler, 1959). At present, quantitative analysis of scaferrin in semen samples is being carried out to examine the possibility that this antigen may be linked to infertility. This work will be presented in a subsequent communication.

\section{This work was supported by a grant from The Population Council.}

\section{REFERENCES}

Hekman, A. \& Rumke, P. (1968) The antigens of human seminal plasma. Fert. Steril. (In press).

Masson, P. L. \& Heremans, J. F. (1966) Studies on lactoferrin, the iron-binding protein of secretions. Protides biol. Fluids, 14, 115.

Szuchet-Derechin, S. \& Johnson, P. (1966) The "albumin" fraction of bovine milk. III. The microheterogeneity of the red protein. Eur. Polym. F. 2, 29.

WerL, A. J. (1965) The spermatozoa-coating antigen (SCA) of the seminal vesicle. Ann. N.Y. Acad. Sci. $124,267$.

WeiL, A. J. (1967) Antigens of the seminal plasma. F. Reprod. Fert., Suppl. 2, 25.

Weil, A. J. \& Finkler, A. E. (1959) Isoantigenicity of rabbit semen. Proc. Soc. exp. Biol. Med. 102, 624.

Weil, A. J., Kotsevalov, O. \& Wirson, L. (1956) Antigens of human seminal plasma. Proc. Soc. exp. Biol. Med. 92, 606.

WeIL, A. J. \& RodenBURG, J. M. (1960) Immunological differentiation of human testicular (spermatocele) and seminal spermatozoa. Proc. Soc. exp. Biol. Med. 105, 43.

WeiL, A. J. \& Rodenburg, J. M. (1962) The seminal vesicle as the source of the spermatozoa-coating antigen of seminal plasma. Proc. Soc. exp. Biol. Med. 109, 567. 